\title{
Injective modules and soluble groups satisfying the minimal condition for normal subgroups
}

\author{
B. Hartley and D. McDougall
}

Let $p$ be a prime and let $Q$ be a centre-by-finite $p^{\prime}$-group. It is shown that the $Z Q$-modules which satisfy the minimal condition on submodules and have p-groups as their underlying additive groups can be classified in terms of the irreducible $Z_{p} Q$-modules. If such a $Z Q$-module $V$ is indecomposable it is either the ZQ-injective hull $W^{\cdot}$ of an irreducible $Z_{p} Q$-module (viewed as a $Z Q$-module) or is the submodule $W\left[p^{n}\right]$ of such a $W$ consisting of the elements $w \in W$ which satisfy $p^{n} w=0$. This classification is used to classify certain abelian-by-nilpotent groups which satisfy Min-n, the minimal condition on normal subgroups. Among the groups to which our classification applies are all quasi-radicable metabelian groups with Min-n, and all metabelian groups which satisfy Min-n and have abelian Sylow $p$-subgroups for all $p$.

It is also shown that if $Q$ is any countable locally finite $p^{\prime}$-group and $V$ is a $Z Q$-module whose additive group is a $p$-group, then $V$ can be embedded in a $Z Q$-module $\bar{V}$ whose additive group is a minimal divisible group containing that of $V$. Some applications of this result are given.

Received 15 September 1970. 


\section{Introduction}

A group $G$ is said to be quasi-radicable if, for each integer $n>0, G$ is generated by the $n$-th powers of its elements. One of the main purposes of this paper is to classify quasi-radicable metabelian groups satisfying Min- $n$, the minimal condition on normal subgroups. However it turns out to be equally convenient to work with a somewhat larger class, namely the class $\underline{\underline{z}}$ of all abelian-by-nilpotent groups which satisfy Min- $n$ and in addition satisfy the condition

(z) If $G \in \underline{\underline{Z}}$ and $P$ is a p-subgroup of $G$ then $\underline{\underline{N}} \cap P$ is contained in the centre of $P$.

Here $G$ denotes the uniquely determined normal subgroup $K$ of $G$ which is minimal subject to the condition that $G / K$ is nilpotent; its existence is assured by Min-n. By a theorem of Baer [4] soluble groups satisfying Min-n, and hence Z-groups, are locally finite; another theorem of Baer [3] states that nilpotent groups with Min-n are centre-by-finite, so that $\underline{\underline{Z}}$-groups are metabelian-by-finite. $\underline{\underline{Z}}$ contains all metabelian groups which satisfy Min-n and have abelian Sylow p-subgroups for all primes $p$; hence ([14], Corollary 3.3) it contains all quasi-radicable metabelian groups with Min-n .

Let $G \in \underline{\underline{Z}}$ and let $K=G \underline{\underline{N}}$. Then $G$ splits over $K$ (Lemma 4.1)

$$
G=K A, K \cap A=1 \text {. }
$$

Here, by the remarks above, $A$ is nilpotent and centre-by-finite; the results of Baer [3] also show that $A$ satisfies Min, the minimal condition on subgroups. Now the condition $(z)$ ensures that the sylow $p$-subgroup of $A$ centralizes the Sylow p-subgroup $K_{p}$ of $K$; hence $K_{p}$ is effectively a module for $A_{p^{\prime}}$, the sylow $p^{\prime}$-subgroup of $A$, satisfying the minimal condition on submodules.

our problem is thus closely related to that of classifying those modules over the integral group ring $Z Q$ of a centre-by-finite $p^{\prime}$-group $Q$ which satisfy Min-Q, the minimal condition on $Q$-submodules, and have $p$-groups as their underlying additive groups. Modules with the latter property will be called p-modules. We shall deal with this problem in 
\$2. Strictly speaking our results simply reduce the classification problem to that of classifying the irreducible $Z_{p} Q$-modules; in the case when $Q$ is abelian this can be done quite easily (Lemma 2.5). We shall see that the indecomposable $p$-modules over $Z Q$ with Min-Q arise naturally from the injective hulls of the irreducible ones, and our results in $\$ 2$ lean heavily on the properties of the injective hull of a module as defined by Eckmann and Schopf [8] (see also [7], p. 384 et seq., or $[15])$.

A problem which arises naturally from the work in $\$ 2$ is the following: Let $V$ be a $Z G$-module, where $G$ is any group, and let $\bar{V}$ be a minimal divisible group containing the additive group $V^{+}$of $V$, or in other words a Z-injective hull of $V^{+}$. Under what conditions can the $Z G$-structure of $V$ be extended to $\bar{V}$ ?

This cannot invariably be done; for example, since a group of type $C_{\infty}$ has no automorphism of order $p$ if $p$ is odd, it cannot be done if $p^{\infty}$

$V$ is a cyclic group of order $p^{2}$ which is a non-trivial module for a cyclic group of odd prime order $p$. In $\$ 3$ we deduce easily from the results of $\$ 2$ that the extension can be carried out provided $V$ is a $p$-module and $G$ is a countable locally finite $p^{\prime}$-group (Theorem Bl). This result is used to construct examples of soluble groups of any given derived length which satisfy Min-n and have a series of finite length in which all the factors are divisible abelian groups (Lema 3.4). These groups are PQ-groups in the sense of [13].

Finally in $\$ 4$ we deduce our classification of Z-groups from the results of $\$ 2$.

We are indebted to $\mathrm{Dr}$ M.C.R. Butler who suggested the possibility of applying the theory of injective modules to the problems described above; this resulted in a considerable simplification of our previous work.

\section{Injective p-modules for centre-by-finite $p^{\prime}$-groups}

We begin by recalling the basic facts about injective modules which we shall need ( $c f$. [7], p. 384 et seq., or [15]). Let $R$ be a ring with 1 . By an $R$-module we shall always understand a right $R$-module on which 
$I$ acts as the identity map. An $R$-module $X$ is called injective if whenever $U \leq W$ are $R$-submodules then every $R$-homomorphism of $U$ into $X$ can be extended to $W$. This is equivalent (but not immediately) to the requirement that $X$ be a direct summand of every $R$-module which contains it. If $V$ is an arbitrary $R$-module then an injective hull of $V$ (in the category of $R$-modules) is an $R$-module $\bar{V}$ satisfying:-

(i) $\vec{V}$ is injective, and either

(ii) no proper submodule of $\bar{V}$ containing $V$ is injective, or

(ii)' $\bar{V}$ is an essential extension of $V$.

Here a module $W$ is said to be an essential (or related) extension of a submodule $U$ if every non-trivial submodule of $W$ meets $U$ non-trivially. It was shown by Eckmann and Schopf [8] that every $R$-module $V$ has an injective hull $\bar{V}$ which is unique in the sense that if $V^{*}$ is another injective hull of $V$ then there is an isomorphism from $\bar{V}$ to $V^{*}$ extending the identity map on $V$.

We shall need the following fact:

LEMMA 2.1. Let $R$ be a ring with 1 , let $V$ be an $R$-module and let $\bar{V}$ be an injective hulz of $V$. Suppose $V=\underset{\lambda \in \Lambda}{\oplus} V_{\lambda}$ where each $V_{\lambda}$ is an R-submoduze of $V$. If either

(i) $\Lambda$ is finite, or

(ii) $R$ satisfies the maximal condition on right ideals, then $\bar{V}=\underset{\lambda \in \Lambda}{\oplus} \bar{V}_{\lambda}$, where $\bar{V}_{\lambda}$ is an injective hull of $V_{\lambda}$.

Proof. We can embed $V$ in a module $W=\underset{\lambda \in \Lambda}{\oplus} W_{\lambda}$, where each $W_{\lambda}$ is an injective hull of $V_{\lambda}$; it will suffice to show that $W$ is an injective hull of $V$. Now $W$ is injective ([15], Theorems 5 and 6) and so we need only show that $W$ is an essential extension of $V$. If this is not the case then there is a non-zero element $w$ of $W$ such that whenever $r \in R$ and $w r \in V$ then $w r=0$. We may express $w$ in the form $w=w_{\lambda_{1}}+\ldots+w_{\lambda_{k}}\left(0 \neq w_{\lambda_{i}} \in w_{\lambda_{i}}, \quad \lambda_{i} \neq \lambda_{j}\right.$ if $\left.i \neq j\right)$ and suppose $k$ is minimal with respect to $w$ having the desired property. 
Now $0 \neq w_{\lambda_{1}}{ }^{r} \in V_{\lambda_{1}}$ for some $r \in R$. Consider $\left(w-w_{\lambda_{1}}\right) r$. If it is zero then $w r=w_{\lambda_{1}}{ }^{r}$, a non-zero element of $V$. Hence $\left(w-w_{\lambda_{1}}\right) r \neq 0$, and by the minimality of $k$ we have $0 \neq\left(w-w_{\lambda_{1}}\right) r s \in V$ for some $s \in R$. Hence $0 \neq w r s=\left(w-w_{\lambda_{1}}\right) r s+w_{\lambda_{1}} r s \in V$, which is a contradiction.

Notice that Lema 2.1 holds in particular when $R$ is the integral group ring of a finite group.

Now it is not difficult to see that every injective $R$-module $U$ is divisible in the sense that $U d=U$ for every element $d$ of $R$ which is not a zero-divisor (of. [11], Theorem 3.1). We shall call an R-module $V$ Z-divisible if the additive group $V^{+}$of $V$ is a divisible group. We then have immediately

\section{LEMMA 2.2. Every injective ZG-module is Z-divisible.}

We shall now see that under certain circumstances the converse is true, and that sometimes the injective hull $\bar{V}$ of a $Z G$-module $V$ has for its additive group a minimal divisible group containing $V^{+}$. This cannot happen when $V$ is cyclic of order $p^{2}$ and $G$ is cyclic of odd prime order $p$, as we have already remarked. Furthermore if $G$ is any infinite group, $D$ is any non-trivial divisible group, $\bar{D}$ is the base-group of the restricted wreath product $D$ wr $G$, and $V=[\bar{D}, G]$, then $V$ is $Z$-divisible but is not $Z G$-injective since it is not complemented by a $Z G$-submodule of $\bar{D}$. Thus in this case the injective hull of $V$ does not have for its additive group a minimal divisible group containing $V^{+}$.

If $p$ denotes a prime (as it always will) and $V$ is any abelian group we denote by $V\left[p^{k}\right]$ the set of elements $v \in V$ satisfying $p^{k} v=0$ (where $k>0$ is an integer). If $V$ is in addition an $R$-module then $v\left[p^{k}\right]$ will be an $R$-submodule of $V$.

LEMMA 2.3. Let $Q$ be a centre-by-finite $p^{\prime}$-group and let $V$ be $a$ p-module over $Z Q$. Suppose that either

(i) $Q$ is finite, or

(ii) $V$ satisfies Min-Q. 
Let $\bar{V}$ be an injective huzz of $V$. Then

(a) $\bar{V}$ is a p-module and $\bar{V}[p]=V[p]$,

(b) $V$ is injective if and only if $V$ is Z-divisible.

For the proof we shall require the following lemma, which is a straightforward consequence of a result of Kovács and Newman [12]. We shall call a module monolithic if the intersection of its non-zero submodules is again non-zero.

LEMMA 2.4. Let $Q$ be a centre-by-finite $p^{\prime}$-group, let $V$ be a ZQ-module and let $W$ be a submodule of $V$. Suppose that $W$ is a $p$-module and is the direct sum of finitely many monolithic submodules, and suppose further that $W$ is a direct summand of $V$ as an additive group. Then $W$ is a direct summand of $V$ as a ZQ-moduZe.

Proof of Lemma $2.3(a)$. In case (i) it is clear that every element of $V[p]$ lies in a finite submodule of $V[p]$. It then follows by Maschke's Theorem that $V[p]$ is generated by its irreducible submodules and so is the direct sum of a selection of them. In case (ii) we find that if $V \neq 0$ then $V[p]$ contains an irreducible submodule, and this is a direct summand of $V[p]$ by Lemma 2.4. By applying this argument to a complementary submodule and continuing in the same way, we find that $V[p]$ is in this case the direct sum of finitely many irreducible submodules. Now $\bar{V}$ is clearly an injective hull of $V[p]$ and so in either case Lemma 2.1 allows us to assume that $V$ is irreducible.

We then have $V \leq \bar{V}[p]$. Since $V$ is certainly a direct summand of the additive group of $\bar{V}[p]$, Lema 2.4 shows that $V$ is a direct summand of the $Z Q$-module $\bar{V}[p]$. But $\bar{V}$ is an essential extension of $V$ and so $V=\bar{V}[p]$.

It follows that the submodule $\bar{V}_{p}$ formed by the p-elements of $\bar{V}$ is monolithic with $V$ as its unique minimal submodule. Now by Lerma 2.2 $\vec{V}_{p}$ is Z-divisible and so it is a direct summand of the additive group of $\bar{V}$. Consequently, by Lemma 2.4 again, $\bar{V}_{p}$ is a direct summand of $\bar{V}$ as ZQ-module. It then follows that $\bar{V}_{p}=\bar{V}$, completing the proof.

(b) If $V$ is injective then it is Z-divisible by Lemma 2.2. 
Conversely suppose that $V$ is Z-divisible, and let $\bar{V}$ be an injective hull of $V$. By $(a)$ the additive group of $\bar{V}$ is a minimal divisible group containing that of $V$, and so $V=\vec{V}$. Hence $V$ is injective.

We conclude this section by describing the structure of $p$-modules with Min- $Q$ over $Z Q$, where $Q$ is a centre-by-finite $p^{\prime}$-group. Let $\left\{V_{\lambda}: \lambda \in \Lambda\right\}$ be a complete set of representatives for the isomorphism types of irreducible $Z_{p} Q$-modules. We view the $V_{\lambda}$ as $Z Q$-modules and denote by $\bar{V}_{\lambda}$ a ZQ-injective hull of $V_{\lambda}$. Let $V_{\lambda}(n)$ denote the submodule of $\bar{V}_{\lambda}$ formed by the elements $v$ satisfying $p^{n} v=0$ $(n=0,1, \ldots)$, and put $V_{\lambda}(\infty)=\bar{V}_{\lambda}$. Then $V_{\lambda}(n)$ is determined up to isomorphism by $\lambda$ and $n$; notice also that by Lemma 2.3 $V_{\lambda}(n+1) / V_{\lambda}(n) \cong V_{\lambda}=V_{\lambda}(1)$, which is irreducible. It follows from this that the $V_{\lambda}(n) \quad(n=0,1, \ldots, \infty)$ are the only submodules of $V_{\lambda}(\infty)$.

THEOREM A. Let $Q$ be a centre-by-finite $p^{\prime}$-group and let $V$ be a p-module over $Z Q$. Then $V$ satisfies Min-Q if and only if $V$ is a direct sum of finitely many submodules each isomorphic to some $V_{\lambda}(n)$ $(1 \leq n \leq \infty)$.

If $V$ satisfies Min-Q and $V$ is expressed in two ways as the direct sum of indecomposable submodules, then there is an automorphism of $V$ mopping the first decomposition onto the second.

since $V_{\lambda}(n)$ is isomorphic to $V_{\mu}(m)$ if and only if $\lambda=\mu$ and $m=n$ it follows that the $p$-modules over $Z Q$ with Min-Q are classified by the functions of finite support from the set of pairs $(\lambda, n)$ $(\lambda \in \Lambda, \quad 1 \leq n \leq \infty)$ to the non-negative integers.

Proof of Theorem A. From our remarks preceding the statement of Theorem A it follows that each $V_{\lambda}(n)$ satisfies Min-Q; hence any finite direct sum of such modules also satisfies Min- $Q$.

Conversely suppose that $V$ satisfies Min-Q. If $V$ is not expressible as stated then among the submodules of $V$ which are not so expressible there is a minimal one. It thus suffices for the proof to 
assume that, while every proper submodule of $V$ is expressible in the manner stated, $V$ itself is not, and to obtain a contradiction. This assumption implies that $V$ is indecomposable.

Let $W$ be the maximal divisible subgroup of $V$ and suppose first that $W \neq 0$. Then $W$ is a submodule of $V$, and it follows from Lemma 2.3 that $W$ is injective. Consequently $W$ is a direct summand of $V$ and so $V=W$. However Lemma 2.4 shows that $W[p]$ is the direct sum of finitely many irreducibles, and Lemma 2.1 then shows that $W$ is the direct sum of the injective hulls of these irreducibles. Therefore $V$ is a direct sum of submodules of type $V_{\lambda}(\infty)$, which is a contradiction. We therefore have that $W=0$.

By the minimal condition the chain $V \geq p V \geq p^{2} V \geq \ldots$ must become stationary after finitely many steps. Since $V$ contains no non-trivial divisible subgroup it follows that $p^{n} V=0$ for some $n$, which we suppose chosen as small as possible. Then $p^{n-1} V \neq 0$ and so $p^{n-1} V$ contains an irreducible submodule $U$. There is an isomorphism of $U$ onto some $V_{\lambda}$, and this may be extended to a homomorphism $\phi$ of $V$ into the injective module $V_{\lambda}(\infty)$. Now $p^{n-1}(V \phi)=\left(p^{n-1} V\right) \phi \neq 0$, and so $V \phi$ has exponent $p^{n}$ precisely. Hence $V \phi=V_{\lambda}(n)$. Let $K$ be the kernel of $\phi$. Then $V / K$, as an additive group, is the direct sum of cyclic groups of the same order $p^{n}$. Such a group is free in the category of abelian groups of exponent dividing $p^{n}$; hence $K$ is a direct summand of $V$ as an additive group. Also, by the minimality of $V, K$ is the direct sum of finitely many submodules of the type $V_{\lambda}(n)$. Since these are all monolithic, Lemma 2.4 shows that $K$ is a direct summand of $V$ as a module. This contradicts the indecomposability of $V$ and establishes the result.

To establish the final statement if suffices, by a well-known version of the Krull-Schmidt Theorem due to Azumaya [1], to show that in the endomorphism ring of each $V_{\lambda}(n), n \geq 1$, the sum of two non-units is a non-unit. Since $V_{\lambda}(n)$ has no proper submodule isomorphic to itself, 
every such non-unit has a non-trivial kernel, which must contain the unique minimal submodule of $V_{\lambda}(n)$. This makes it clear that the sum of two such non-units is a non-unit, as claimed.

As we have remarked, the irreducible $Z_{p} Q$-modules are quite readily obtained when $Q$ is abelian. In fact let $Q$ be any periodic abelian group, which need not even be a $p^{\prime}$-group for these purposes, and let $k$ be an algebraic closure of $Z_{p}$. Let $\theta$ be a homomorphism of $Q$ into the multiplicative group $k^{*}$ of non-zero elements of $k$. Then since the elements of $Q \theta$ are all roots of unity, it follows that the additive group $L_{\theta}$ generated by $Q \theta$ is in fact a field. Let $K_{\theta}$ be the $Z_{p}{ }^{Q \text {-module }}$ whose underlying vector space is $L_{\theta}$ with the Q-action given by

$$
v g=v \cdot g \theta \quad(v \in V, g \in Q) \text {. }
$$

Since $Q \theta$ generates $L_{\theta}$ additively any $Q$-submodule of $K_{\theta}$ is invariant under multiplication by any element of $L_{\theta}$; consequently $K_{\theta}$ is irreducible. We have the following result, which is no doubt well known.

LEMMA 2.5. With the above notation

(i) every irreducible $\mathrm{Z}_{p} \mathrm{Q-module}$ is isomorphic to some $K_{\theta}$;

(ii) $K_{\theta} \cong K_{\phi}$ if and only if $L_{\theta}=L_{\phi}$ and $\theta=\phi \rho$ for some element $\rho$ of the Gazois group of $L_{\theta}$ over $Z_{p}$.

Proof $(i)$. Let $V$ be an irreducible $Z_{p} Q$-module and let $E=\operatorname{End}_{Q} V$, which is a division algebra over $Z_{p}$ by Schur's Lemma. Since $Q$ is abelian, if $g \in Q$ the map $g \tau$ given by

$$
v(g \tau)=v g \quad(v \in V)
$$

is an element of $E$ and $\tau$ maps $Q$ homomorphically into the centre $Z$ of $E$, which is a field. Let $L$ be the additive subgroup of $E$ generated by $Q \tau$. Then since the elements of $Q \tau$ are roots of unity $L$ is a subring of $Z$ which is algebraic over $Z_{p}$, and so it is a field. 
is an $L$-module and since $Q \tau \leq L$ we must have $\operatorname{dim}_{L} V=1$. So $V=v L$ for some $v \in V$. Choose a monomorphism $\bar{\psi}$ of $L$ into $k$ and define

$$
(v Z) \psi=2 \bar{\psi} \quad(Z \in L) \text {. }
$$

This gives a well-defined additive isomorphism of $V$ onto the field $L \bar{\psi}$. Let $\theta=\tau \bar{\psi}$. Then $\theta$ maps $Q$ homomorphically into the multiplicative group $k^{*}$ and $L \bar{\psi}$ is additively generated by $Q \theta$. We now verify that the map $\psi$ is an isomorphism of $V$ onto $K_{\theta}$. In fact if $u \in V$ and $g \in Q$ we have $u=v l$ for some uniquely determined $z \in L$, and

$$
(u g) \psi=[v L(g \tau)] \psi=(2 . g \tau) \bar{\psi}=\tau \bar{\psi} \cdot g \tau \bar{\psi}=u \psi \cdot g \theta,
$$

as required.

(ii) Suppose first that $L_{\theta}=L_{\phi}$ and $\theta=\phi \rho$ with $\rho$ an element of the Galois group of $L_{\theta}$ over $Z_{p}$. Then $\rho$ is an additive automorphism of $L_{\theta}$, and if $x \in L_{\theta}$ and $g \in Q$ we have

$$
(x \cdot g \phi) \rho=x \rho \cdot g \phi \rho=x \rho \cdot g \theta
$$

so that $\rho$ maps $K_{\phi}$ isomorphically onto $K_{\theta}$.

Suppose conversely that $K_{\phi} \cong K_{\theta}$. Then since the kernel of $\phi$ is the kernel of the representation of $Q$ determined by $K_{\phi}$ we must have that $\theta$ and $\phi$ have the same kernel. Therefore $Q \theta \cong Q \phi$. Now $k^{\star}$ is a direct product of groups of type $C_{q^{\infty}}$, one for each prime $q \neq p$; it follows that no two distinct subgroups of $k^{*}$ are isomorphic. Hence $Q \theta=Q \phi$ and so $L_{\theta}=L_{\phi}$. Let $\rho$ be any isomorphism of $K_{\phi}$ onto $K_{\dot{\theta}}$. Then $\rho$ is an additive automorphism of $L_{\theta}$ and, since multiplication by any non-zero element of $L_{\theta}$ determines an automorphism of $K_{\theta}$, we may choose $\rho$ so that $1 \rho=1$. Then for $x \in K_{\phi}$ and $g \in Q$ we have

$$
(x \cdot g \phi) \rho=x \rho \cdot g \theta \text {. }
$$

Putting $x=1$ gives $\phi \rho=\theta$ and so $(x . g \phi) \rho=x \rho . g \phi \rho$, or $(x . y) \rho=x \rho . y \rho$ for $x \in L_{\theta}$ and $y \in Q \theta$. Since $Q \theta$ generates $L_{\theta}$ additively it follows that $\rho$ preserves multiplication, and so belongs to 
the Galois group of $L_{\theta}$ over $Z_{p}$.

\section{Embedding in Z-divisible modules}

It follows in particular from the existence of the injective hull, that every $R$-module can be embedded in an injective $R$-module. In fact this was first proved by Baer [2]. Lemma 2.2 then gives

LEMMA 3.1. Every ZG-module can be embedded in a Z-divisible ZG-moduze.

It is natural to ask under what circumstances a $Z G$-module $V$ may be embedded in a Z-divisible $Z G$-module $\bar{V}$ whose additive group is a minimal divisible group containing that of $V$. This is not always possible, as we remarked in $\$ 1$. Now the following facts are immediate from Lemma 2.3:

LEMMA 3.2. Let $Q$ be a centre-by-finite $p^{\prime}$-group and let $V$ be a p-module over $\mathrm{ZQ}$. Let $\bar{V}$ be a minimal divisible group containing the additive group of $V$, and suppose that either

(i) $Q$ is finite, or

(ii) $V$ satisfies Min-Q.

Then

(a) $\bar{V}$ admits a ZQ-module structure extending that on $V$;

(b) if $V_{1}, V_{2}$ are $Z Q$-modules containing $V$ in such a manner that their additive groups are minimal divisible groups containing that of $V$, then the identity map on $V$ extends to an isomorphism of $V_{1}$ onto $V_{2}$.

We shall now show that (a) holds in considerably greater generality:

THEOREM B1. Let $Q$ be a countable locally finite $p^{\prime}$-group, let $V$ be a p-module over $Z Q$, and let $\bar{V}$ be a minimal divisible group containing the additive group of $V$. Then $\bar{V}$ admits a ZQ-module stmucture extending that of $V$.

In this generality, however, (b) of Lemma 3.2 may break down, and the resulting $Z Q$-module $\bar{V}$ is not always even determined up to isomorphism by $V$. We shall not pursue this point at present, but hope to return to it in a later publication. We have no idea whether the restriction of 
countability is necessary.

$$
\begin{aligned}
& \text { Proof. Write } Q=\bigcup_{i=0}^{\infty} Q_{i} \text {, where } \\
& \qquad 1=Q_{0} \leq Q_{1} \leq \ldots
\end{aligned}
$$

is a tower of finite subgroups of $Q$. We shall construct for each $n \geq 0$ a map $f_{n}: \bar{V} \times Z Q_{n} \rightarrow \bar{V}$ which makes $\bar{V}$ into a $Z Q_{n}$-module and is such that

$$
(v, r) f_{n}=v r \quad\left(v \in V, r \in Z_{n}\right)
$$

We shall also arrange that each $f_{n+1}$ extends $f_{n} \cdot$ These maps will then determine a map from $\bar{V} \times Z Q$ to $\bar{V}$ which makes $\bar{V}$ into a $Z Q$-module in the required manner.

Now $f_{0}$ can certainly be obtained (and is in fact uniquely determined). Suppose that for some $n \geq 0, f_{n}$ has been constructed. It follows from Lemma 3.2 that there is a $Z$-divisible $Z Q_{n+1}$-module $W$ containing the restricted module $V_{Q_{n+1}}$ in such a manner that the additive group of $W$ is a minimal divisible group containing the additive group of $V_{Q_{n+1}}$ (or the additive group of $V$, which is the same thing). It further follows from Lemma 3.2 that the identity map on $V$ can be extended to a $Z Q_{n}$-isomorphism $\phi$ of the $Z Q_{n}$-module $\left(\bar{V}, f_{n}\right)$ onto $W_{Q_{n}}$. The mapping $f_{n+1}: \bar{V} \times Z Q_{n+1} \rightarrow \bar{V}$ defined by

$$
(v, r) f_{n+1}=(v \phi \cdot r) \phi^{-1} \quad\left(v \in V, r \in Z Q_{n+1}\right)
$$

then makes $\bar{V}$ into a $Z Q_{n+1}$-module, extends $f_{n}$, and satisfies (1) with $n$ replaced by $n+1$. Thus the maps $f_{n}$ can be constructed and the result is established.

THEOREM B2. Let $Q$ be a countable locally finite $p^{\prime}$-group and let $V$ be a p-module over $Z Q$ satisfying Min-Q. Then $V$ can be embedded in a Z-divisible p-module over $Z Q$ which satisfies Min-Q. 
Proof. Let $\bar{V}$ be a $Z Q$-module containing $V$ in such a manner that the additive group of $\bar{V}$ is a minimal divisible group containing that of $V$. The existence of such a $\bar{V}$ is given by Theorem B1. Then $\bar{V}[p]=V[p]$ and Theorem B2 follows from the following lemma:

LEMMA 3.3. Let $V$ be an abelian p-group admitting a set $\Omega$ of distributive operators. Then $V$ satisfies Min $\Omega$, the minimal condition on $\Omega$-subgroups, if and only if $V[p]$ satisfies Min- $\Omega$.

Proof. It is clear that if $V$ satisfies Min- $\Omega$ so does $V[p]$. Conversely assume that $V[p]$ satisfies $\operatorname{Min}-\Omega$ and let

$$
V_{1} \geq V_{2} \geq \cdots
$$

be a descending chain of $\Omega$-subgroups of $V$. Consider the $\Omega$-subgroups

$$
U_{i, m}=p^{m}\left(V_{i} \cap v\left[p^{m+1}\right]\right) \quad(i=1,2, \ldots ; m=0,1, \ldots)
$$

of $V[p]$. Clearly $U_{i, m} \geq U_{i+1, m}$ and $U_{i, m} \geq U_{i, m+1}$. Therefore $U_{i, m} \geq U_{j, n}$ if $j \geq i$ and $n \geq m$, and since $V[p]$ satisfies Min- $\Omega$ we can choose $i$ and $m$ so that

$$
U_{i, m}=U_{j, n} \text { for } j \geq i \text { and } n \geq m \text {. }
$$

Now the map $v \rightarrow p^{k} v$ determines an embedding of $V\left[p^{k+1}\right] / V\left[p^{k}\right]$ in $V[p]$ and so each $V\left[p^{k+1}\right] / V\left[p^{k}\right]$ satisfies Min- . Therefore $V\left[p^{k}\right]$ satisfies $\operatorname{Min} \Omega$ and we may suppose $i$ chosen in (2) so that in addition

$$
V_{i} \cap V\left[p^{m+1}\right]=V_{j} \cap V\left[p^{m+1}\right] \text { whenever } j \geq i \text {. }
$$

We now show by induction on in that

$$
V_{i} \cap V\left[p^{n}\right]=V_{j} \cap V\left[p^{n}\right] \text { for all } j \geq i \text { and } n \geq m+1
$$

Indeed suppose (3) holds for some $n \geq m+1$ and let $v \in v_{i} \cap v\left[p^{n+1}\right]$. Then by (2) $p^{n} v \in U_{i, n}=U_{j, n}$ and so $p^{n} v=p^{n} w$ for some $w \in V_{j} \cap v\left[p^{n+1}\right]$. Therefore $p^{n}(v-w)=0$ and $v-w \in V_{i} \cap V\left[p^{n}\right]=V_{j} \cap V\left[p^{n}\right]$. Hence $v \in V_{j} ;$ as required. 
It follows from (3), and the fact that $V=\underset{n \geq m+1}{U} V\left[p^{n}\right]$, that
$V_{i}=V_{j}$ for all $j \geq i$, whence we have that $V$ has Min- $\Omega$.
In [13] a group possessing a series of finite length in which the factors are periodic divisible abelian groups was called a PQ-group. We extend Example 4 of [14] as follows:-

LEMMA 3.4. The class of PQ-groups satisfying Min-n contains groups of any prescribed derived length.

Proof. The construction is similar to that of [14]. Suppose that we have constructed, for some integer $n \geq 1$, a PQ-group $G_{n}$ which satisfies Min-n, is a $\pi$-group for some finite set $\pi$ of primes, and is in addition monolithic with monolith $M$. As $G_{1}$ we may take a group of type $C_{\infty}$, where $q$ is a prime. Let $x$ be an element of prime order $p \notin \pi$, , and let $X$ be the base group of $\langle x\rangle$ wr $G_{n}=W$. There is a chief series of $W$ through $X$. If $M$ centralized every factor below $X$ in this series it would centralize $X$ itself since $X$ is a p-group and $p \notin \pi$. Consequently $M$ fails to centralize some such factor, which then furnishes a faithful irreducible p-module $V$ for $G_{n}$. It follows from Theorem Bl and Lemma 3.3 that there is a p-module $\bar{V}$ for $G_{n}$ which satisfies $M i n-G_{n}$ and is such that $V=\bar{V}[p]$. Let $G_{n+1}$ be the semidirect product $\bar{V} G_{n}$. Then $G_{n+1}$ is a $\underline{\underline{Q}}$ group with Min-n and is monolithic with monolith $V$. It follows easily that $G_{n+1}$ has derived length $n+1$ exactly.

\section{Classification of $\underline{Z}$-groups}

our aim in this section is to classify, up to isomorphism, groups in the class $\underline{\underline{Z}}$, the class introduced in $\S 1$. We shall classify these groups in terms of nilpotent centre-by-finite groups with Min and irreducible modules for such groups. A result which will be of fundamental importance for our classification is the following:

LEMMA 4.1. Let $G \in \underline{\underline{Z}}$. Then $G$ splits over $G^{\mathbb{N}}$ and the 
complements to $G \stackrel{\mathbb{N}}{\underline{N}}$ are conjugate in $G . G / G \stackrel{\mathbb{N}}{\underline{N}}$ is centre-by-finite.

Our proof of Lemma 4.1 will depend on properties of the class $\underline{\underline{U}}$ introduced in [9]. We recall that a locally finite group $X$ belongs to $\underline{\underline{U}}$ if and only if $X$ has a series of finite length with locally nilpotent factors and every subgroup of $X$ has conjugate sylow (that is, maximal) $\pi$-subgroups for all sets $\pi$ of primes.

LEMMA 4.2. Let $G$ be a soluble group satisfying Min-n. Suppose that $G$ contains a normal subgroup of finite index which is - nilpotent-by-locally nilpotent. Then $G \in \underline{\underline{\mathrm{U}}}$.

Proof. By Baer's Theorem [4] $G$ is locally finite. Since the condition Min-n is inherited by normal subgroups of finite index [16], and since the class $\underline{\underline{U}}$ is closed under extensions by finite soluble groups ([10], Lemma 6.6) we may assume that $G$ contains a normal nilpotent subgroup $H$ such that $G / H$ is locally nilpotent. Then $G / H$ is a locally nilpotent group satisfying Min-n. Such groups satisfy the minimal condition on all subgroups ([6], Corollary 4.6) and so are countable and abelian-by-finite. Therefore, arguing as before, we may suppose that $G / H$ is abelian.

Let $X$ be any subgroup of $G$ and let $K$ be the Sylow $\pi$-subgroup of $H$. Then $X K / K \cong X / X \cap K$ and, since $X \cap K$ is a normal $\pi$-subgroup of $X$, the conjugacy of the Sylow $\pi$-subgroups of $X K / K$ implies the conjugacy of those of $X$. We may therefore assume that $K=1$.

Let $S$ and $T$ be Sylow $\pi$-subgroups of $X$. We shall now show that $S$ and $T$ are conjugate in $X$ by induction on the nilpotency class $c$ of $H$. If $c=0$ then $H=1, X$ is abelian, and $S=T$. Assume then that $c>0$ and let $Z$ be the centre of $H$. We may assume by induction that $X$ contains an element $x$ such that $\left\langle S^{x} Z / Z, T Z / Z\right\rangle$ is a $\pi$-group, $U / Z$ say. Now $U / Z$ must be countable and so $U=2 W$ for some $\pi$-subgroup $W$ of $U$ (see for instance [10], Lemma 2.1). Let $L$ be any subgroup of $W$ and let $F$ be any finite subgroup of $L$. Then $C_{Z}(F)=Z \cap C_{G}(H F)$, which is normal in $G$ since $G / H$ is abelian. Therefore by Min-n we crn choose $F$ so that $C_{Z}(F)$ is minimal among the centralizers in $Z$ of the finite subgroups of $L$. Then clearly 
$C_{Z}(F)=C_{Z}(L)$. It follows from Lemma 4.3 of [10] that every countable subgroup of $U$ containing $W$ has conjugate Sylow $\pi$-subgroups, and hence from Theorem B of [10] that every countable subgroup whatsoever of $U$ has conjugate sylow $\pi$-subgroups. Therefore $S^{x}$ and $T$ are conjugate in the group they generate, which establishes the lemma.

Proof of Lemma 4.1. We have by Lemma 4.2 that $G \in \underline{\underline{U}}$. Let $K$ be the uniquely determined normal subgroup of $G$ which is minimal subject to the condition that $G / K$ is locally nilpotent. Then $K$ is abelian. By [9], Theorem 4.12, $G$ splits over $K$ and the complements to $K$ in $G$ are conjugate - in fact they are the basis normalizers of $G$. We shall show that $K=N$. Now it is clear from (2) (in $\S 1$ ) that every $p$-subgroup of $G$ is nilpotent. Since $G / K$ satisfies $M i n$, as we have seen, and is therefore countable, every $p$-subgroup of $G / K$ is the image of a $p$-subgroup of $G$ ([10], Lemma 2.1). Therefore the Sylow $p$-subgroups of $G / K$ are nilpotent and so $G / K$ is nilpotent. Hence $K=\stackrel{\underline{N}}{\stackrel{N}{N}}$.

Finally, since it is a nilpotent group satisfying Min-n, $G / K$ is centre-by-finite by a theorem of Baer [3].

Lemmas 4.1 and 4.2 are generalizations of Theorem 3.5 and 5.6 of [14].

Now it follows from Lemma 4.1 that in trying to classify groups in the class $\underline{\underline{Z}}$ it is sufficient to restrict ourselves to considering those groups $G \in \underline{\underline{Z}}$ such that $G / G^{\mathbb{N}}$ is isomorphic to a given nilpotent centre-by-finite group $A$ satisfying the minimal condition.

Let $A_{p}$, be the sylow $p^{\prime}$-subgroup of $A$ and let $\left\{V_{\lambda} ; \lambda \in \Lambda_{p}\right\}$ be a complete set of representatives for the isomorphism classes of non-trivial irreducible $Z_{p} A_{p}$-modules. We assume the sets $\Lambda_{p}$ to be pairwise disjoint, as we may. Notice that if $A$ is actually abelian then the $V_{\lambda}$ may be constructed by the method of Lemma 2.5. For $n=1,2, \ldots, \infty$ let $V_{\lambda}(n)$ denote the $A_{p^{\prime}}$-module obtained from $V_{\lambda}$ as described before Theorem $A$, and view each $V_{\lambda}(n)$ as an $A$-module by 
allowing $A_{p}$ to act trivially. Let $\Lambda=\cup_{p} \Lambda_{p}$ and let $\mathrm{X}=\mathrm{X}(A)$ denote the set of all external direct sums of finitely many modules $V_{\lambda}(n)$ $(n=1,2, \ldots, \infty ; \lambda \in \Lambda)$. We admit a zero module to $X$ as the direct sum of the empty set.

An equivalence relation is introduced on $X$ as follows. First, if $X \in X$ and $\alpha \in$ Aut $A$, let $X^{\alpha}$ be the $A$-module which has $X$ as its underlying additive group and which has the A-action defined by

$$
(x, a) \rightarrow x(a \alpha) \quad(x \in X, a \in A) \text {. }
$$

Now if $X$ and $Y$ are elements of $X$ we define $X \sim Y$ to mean that $X \cong Y^{\alpha}$ for some $\alpha \in$ AutA; we shall say that $X$ is an automorphism conjugate of $Y$. The relation of automorphism conjugacy is easily seen to be an equivalence relation on $X$.

Finally for each $X \in X$ let $X A$ denote the semidirect product of $X$ by $A$, that is the group consisting of all pairs $(x, a)$, where $x \in X$, $a \in A$, with the multiplication $(x, a)\left(x^{\prime}, a^{\prime}\right)=\left(x+x^{\prime} a^{-1}, a a^{\prime}\right)$. When appropriate we shall identify $X$ with a subgroup of $X A$ in the usual manner. We now have

THEOREM C. With the above notation, if $X \in X$ then $H=X A \in \underline{\underline{z}}$,

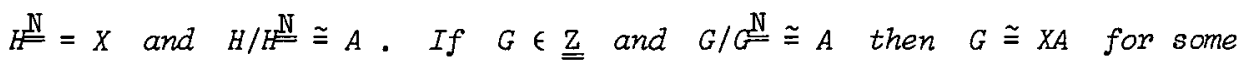
$X \in X$. If $X, Y \in X$ then $X A \cong X A$ if and only if $X \sim Y$.

Thus there is a natural one-to-one correspondence between the isomorphism classes of groups $G$ in $\underline{\underline{Z}}$ with $G / G \underline{\mathbb{N}} \cong A$, and the automorphism conjugacy classes of elements of $X$. We shall have more to say about the relation of automorphism conjugacy after proving Theorem $C$.

Notice that, with the notation of Theorem $\mathrm{C}, H$ will be quasi-radicable if and only if $A$ is quasi-radicable. For if $A$ is quasi-radicable and $n>1$ then the subgroup generated by the $n$-th powers of elements of $H$ contains $A$ and so, being normal in $H$, contains $[X, A]=[H \stackrel{N}{N}, A]=H \stackrel{N}{N}=X$. Since $A$ is in any case centre-by-finite it follows that $H$ is quasi-radicable if and only if $A$ is abelian and radicable (that is, every element has an $n$-th root for all $n>1$ ). 
Proof of Theorem $C$. If $V_{\lambda}$ is any irreducible module in $X$ then since $V_{\lambda}$ is non-trivial and irreducible the submodule $\left[V_{\lambda}, A\right]$ additively generated by the elements $v-v a\left(v \in V_{\lambda}, a \in A\right)$ must be the whole of $V_{\lambda}$. It then follows easily, since $V_{\lambda}(n+1) / V_{\lambda}(n) \cong V_{\lambda}$ if $n$ is finite, that $\left[V_{\lambda}(m), A\right]=V_{\lambda}(m)$ for any $m=1,2, \ldots, \infty$. Hence $[X, A]=X$ for any $X \in X$. Since $X A / X \cong A$, which is nilpotent, this means that $X=(X A) \stackrel{\text { N }}{=}$.

Now let $G \in \underline{\underline{Z}}$ and suppose that $G / G^{\mathbb{N}} \cong A$. If $G=1$ then $G \cong A$ and taking $X$ to be the zero module there is nothing to prove. Thus we may assume that $G \stackrel{N}{\underline{N}} \neq 1$. Then by Lemma 4.1 we have, if $K=G \stackrel{N}{\underline{N}}$

$$
G=\overline{K A}, \quad K \cap \bar{A}=1
$$

for some $\bar{A} \cong A . G$ is locally finite and the condition ( $\mathrm{Z}$ ) ensures that the Sylow $p$-subgroup $\bar{A}_{p}$ of $\bar{A}$ centralizes the Sylow $p$-subgroup $K_{p}$ of the abelian group $K$. Therefore if we view $K_{p}$ as an $\bar{A}_{p}{ }^{\prime-m o d u l e}$ in the natural way it satisfies $\operatorname{Min}-\bar{A}_{p}$. By Lemma $4.1 \bar{A}_{p^{\prime}}$ is centre-by-finite and so by Theorem $A K_{p}$ is a direct sum of finitely many submodules of the type $W(n)$, where $W$ is some irreducible $Z_{p} \bar{A}_{p}$-module. Now since $K$ is a non-trivial abelian group it follows from (4) that $K=[K, \bar{A}]$. Hence $K_{p}=\left[K_{p}, \bar{A}_{p^{\prime}}\right]$ and

$$
W(n)=\left[W(n), \bar{A}_{p^{\prime}}\right] \text {. }
$$

Consequently $W$ must not be a trivial module. Otherwise, since $W$ determines $W(n)$ up to isomorphism $W(n)$ would be trivial, in contradiction to (5).

Let $a \rightarrow \bar{a}$ be an isomorphism of $A$ onto $\bar{A}$. We view $K$ as an $A$-module by defining

$$
x a=\bar{a}^{-1} x \bar{a} \quad(x \in K, \quad a \in A) .
$$

It now follows from the remarks just made that $K$ is isomorphic to some module $X \in X$. Let $\psi$ be an $A$-isomorphism of $X$ onto $K$. Then the 
map $(x, a) \rightarrow x \psi \cdot \bar{a}$ maps $X A$ isomorphically onto $G$.

Finally let $X, Y \in X$. If $X \sim Y$ then $X \cong Y^{\alpha}$ for some $\alpha \in$ Aut $A$. Let $\psi$ be an $A$-isomorphism of $X$ onto $Y^{\alpha}$. Then the map $(x, a) \rightarrow(x \psi, a \alpha)$ maps $X A$ isomorphically onto $Y A$. On the other hand suppose that $X A \cong Y A$ and let $\phi$ be an isomorphism of $X A$ onto $Y A$. We have already seen that $X=(X A) \stackrel{\underline{N}}{\underline{N}}$ and $Y=\langle Y A)^{\underline{N}}$ and so $\phi$ maps $X$ onto $Y$. Since $Y$ is abelian the complements to it in $Y A$ are conjugate under the automorphism group of $Y_{A}$; in fact in this case, by Lemma 4.I, they are conjugate in $Y A$ itself. We may therefore assume that $\phi$ maps the elements of the form $(0, a)$ in $X A$ to the elements of similar form in $Y A$. Thus $\phi$ has the form $(x, a) \rightarrow(x \psi, a \alpha)$ where $\psi$ is an additive isomorphism of $X$ onto $Y$ and $\alpha$ is a bijection of $A$ onto itself. It is then easy to verify that $\alpha \in \operatorname{Aut} A$ and $\psi$ determines an isomorphism of $X$ onto $y^{\alpha}$. Therefore $X \sim Y$, which completes the proof of Theorem $C$.

We notice, for example, that if $A$ is a non-trivial locally cyclic $p^{\prime}$-group then there is, up to automorphism conjugacy, exactly one faithful irreducible $Z_{p} A$-module. For by Lemma 2.5 every such module has the form $K_{\theta}$ for some monomorphism $\theta$ of $A$ into an algebraic closure $k$ of $Z_{p}$. The existence of such a $\theta$ follows since $A$ is a locally cyclic $p^{\prime}$-group, and clearly $K_{\phi}=K_{\theta}^{\phi \theta^{-1}}$. Consequently there is, up to isomorphism, exactly one quasi-radicable metabelian group with Min-n of the form $N A$, where $N$ is a normal p-subgroup faithfully and irreducibly transformed by a given non-trivial radicable locally cyclic $p^{\prime}$-group $A$. Such groups were first constructed by Ċarin [5].

Let $V_{\lambda}(n)^{m}$ denote that member of $X$ which is given as the direct sum of $m$ copies of $V_{\lambda}(n) \quad(m \geq 1)$. Then, since each member $X$ of $X$ is given together with a direct decomposition, $X$ determines uniquely a set 


$$
S(X)=\left\{v_{\lambda_{1}}\left(n_{1}\right)^{m_{1}}, \ldots, v_{\lambda_{k}}\left(n_{k}\right)^{m_{k}}\right\}
$$

where $k \geq 0$ and the pairs $\left(\lambda_{i}, n_{i}\right)$ are all distinct. Now if elements $X$ and $Y$ of $X$ are isomorphic then their $p$-components are isomorphic as $A_{p^{\prime}}$-modules for each prime $p$; Theorem $A$ then shows that this happens if and only if $S(X)=S(Y)$.

LEMMA 4.3. Let $X, Y \in X$ and suppose

$$
S(x)=\left\{v_{\lambda_{1}}\left(n_{1}\right)^{m_{1}}, \ldots, v_{\lambda_{k}}\left(n_{k}\right)^{m} k\right\}
$$

and

$$
S(Y)=\left\{v_{\mu_{1}}\left(s_{1}\right)^{t}, \ldots, v_{\mu_{1}}\left(s_{2}\right)^{t}{ }^{t}\right\}
$$

Then $x \sim y$ if and only if

(i) $k=2$,

(ii) there exists on automorphism $\alpha$ of $A$ and a permutation $\sigma$ of $\{1,2, \ldots, k\}$ such that $V_{\lambda_{i}} \cong V_{\mu \sigma}^{\alpha}, n_{i}=s_{i \sigma}, m_{i}=t_{i \sigma}$ for $1 \leq i \leq k$.

Proof. Suppose first that $X \sim Y$. Then $X \cong y^{\alpha}$ for some $\alpha \in$ AutA and, since $(U \oplus W)^{\alpha}=U^{\alpha} \oplus W^{\alpha}$ for any $A$-modules $U$ and $W$, we have (6) $v_{\lambda_{1}}\left(n_{1}\right)^{m_{1}} \oplus \ldots \oplus v_{\lambda_{k}}\left(n_{k}\right)^{m_{k}} \cong\left(v_{\mu_{1}}\left(s_{1}\right)^{\alpha}\right)^{t_{1}} \oplus \ldots \oplus\left(v_{\mu_{2}}\left(s_{2}\right)^{\alpha}\right)^{t_{2}}$. Since the modules $V_{\lambda_{i}}\left(n_{i}\right)$ and $V_{\mu_{j}}\left(s_{j}\right)^{\alpha}$ are indecomposable it follows from Theorem A that there is a one-to-one correspondence between the summands of this form on the two sides of (6) such that corresponding summands are isomorphic. Now $V_{\mu_{i}}\left(s_{i}\right)^{\alpha} \cong V_{\mu_{j}}\left(s_{j}\right)^{\alpha}$ if and only if $i=j$; consequently to each $i$ with $l \leq i \leq k$ there is a uniquely determined 
integer $i \sigma$ with $1 \leq i \sigma \leq 2$. such that $V_{\lambda_{i}}\left(n_{i}\right) \cong V_{\mu_{i \sigma}}\left(s_{i \sigma}\right)^{\alpha}$. It then follows that $n_{i}=s_{i \sigma}, m_{i}=t_{i \sigma}, k=2$, and that $\sigma$ is a permutation of $\{1,2, \ldots, k\}$. Clearly $V_{\lambda_{i}} \cong V_{\mu_{i \sigma}^{\alpha}}^{\alpha}$.

Conversely suppose that $(i)$ and $(i i)$ hold. Then by Theorem A $V_{\mu_{i \sigma}}\left(s_{i \sigma}\right)^{\alpha}$ must be isomorphic to some module of the form $V_{\lambda}(n)$, and consideration of the minimal submodules shows that the module required must be $V_{\lambda_{i}}\left(n_{i}\right)$. We then easily obtain (6) and hence that $x \cong y^{\alpha}$, as required.

Let us call an abelian p-group homogeneous if it is either homocyclic or divisible. As an application of Theorem C and Lemma 4.3 we prove

COROLLARY 4.4. Let $G_{1}$ and $G_{2}$ belong to $\underline{\underline{z}}$. For $i=1,2$ let $K_{i}=\left(G_{i}\right)^{\mathbb{N}}$, let $N_{i}$ be the product of the minimal normal subgroups of $G_{i}$ contained in $K_{i}$, and let $A_{i}$ be a complement for $K_{i}$ in $G_{i}$. Suppose that, for each prime $p$, the p-component of $K_{i}$ is homogeneous of exponent $p^{n_{i}(p)}$. Then $G_{1} \cong G_{2}$ if and only if

(i) $n_{1}(p)=n_{2}(p)$ for each prime $p$,

(ii) $N_{1} A_{1} \cong N_{2} A_{2}$.

Proof. If $G_{1} \cong G_{2}$ then any isomorphism from $G_{1}$ to $G_{2}$ maps $K_{1}$ onto $K_{2}$, and as in the argument of Theorem $C$ there exists an isomorphism which maps $A_{1}$ onto $A_{2}$. Such an isomorphism maps $N_{1} A_{1}$ isomorphically onto $\mathrm{N}_{2} \mathrm{~A}_{2}$. Thus the necessity of the conditions is clear.

To see the sufficiency we notice first that by Theorem $C$ the minimal normal subgroups of $G_{i}$ in $K_{i}$ are non-central. Hence $N_{i}=\left[N_{i}, A_{i}\right]$, and so $N_{i}=\left(N_{i} A_{i}\right)^{\stackrel{N}{N}}$. Therefore it follows from (ii) that $A_{1} \cong A_{2}$. Theorem $\mathrm{C}$ now allows us to assume that $G_{i}=X_{i} A$, where $A$ is a non-trivial nilpotent centre-by-finite group with Min and $X_{i} \in X=X(A)$ 
$(i=1,2)$. Since the $p$-components of $X_{1}$ are homogeneous,

$$
s\left(x_{1}\right)=\left\{v_{\lambda_{1}}\left(s_{1}\right)^{t_{1}}, \ldots, v_{\lambda_{k}}\left(s_{k}\right)^{t_{k}}\right\}
$$

where $\lambda_{1}, \ldots, \lambda_{k}$ are all distinct and $s_{i}=n_{1}(p)$ if $v_{\lambda_{i}}$ is a p-module. The subgroup $N_{1} A_{1}$ of $G_{1}$ corresponds naturally to $Y_{1} A_{1}$ where $s\left(Y_{1}\right)=\left\{V_{\lambda_{1}}(1)^{t_{1}}, \ldots, V_{\lambda_{k}}(1)^{t_{k}}\right\}$. Thus $k$ is the number of distinct isomorphism types of $A$-modules in $Y_{1}$. It now follows from (ii) that $s\left(x_{2}\right)=\left\{V_{\mu_{1}}\left(u_{1}\right)^{w_{1}}, \ldots, v_{\mu_{k}}\left(u_{k}\right)^{w_{k}}\right\}$, where $\mu_{1}, \ldots, \mu_{k}$ are all distinct. Since $Y_{1} A \cong Y_{2} A$, Theorem $C$ gives $Y_{1} \sim Y_{2}$. Hence by Lemma 4.3 there is an automorphism $\alpha$ of $A$ and a permutation $\sigma$ of $\{1,2, \ldots, k\}$ such that $v_{\lambda_{i}} \cong V_{\mu_{i \sigma}^{\alpha}}^{\alpha}$ and $t_{i}=w_{i \sigma}$. Now $s_{i}=n_{1}(p)$ and $u_{i \sigma}=n_{2}(p)$, where $p$ is the prime such that both $V_{\lambda_{i}}$ and $v_{\mu_{i \sigma}}^{\alpha}$ are $p$-modules. Hence by $(i) s_{i}=u_{i \sigma}$. Lemma 4.3 now shows that $X_{1} \sim X_{2}$, and Theorem $\mathrm{C}$ gives $X_{1} A \cong X_{2} A$, which completes the proof.

\section{References}

[1] Gorô Azumaya, "On generalized semi-primary rings and Krull-Remak-Schmidt's theorem", Japan J. Math. 19 (1948), 525-547.

[2] Reinhold Baer, "Abelian groups that are direct summands of every containing group", Buzz. Amer. Math. Soc. 46 (1940), 800-806.

[3] Reinhold Baer, "Nilgruppen", Math. 2. 62 (1955), 402-437.

[4] Reinhold Baer, "Irreducible groups of automorphisms of abelian groups", Pacific J. Math. 14 (1964), 385-406.

[5] V.S. Carin, "A remark on the minimal condition for subgroups", (Russian), Dokl. Akad. Nauk SSSR 66 (1949), 575-576. 
[6] S.N. Cernikov, "Finiteness conditions in group theory", (Fussian), Uspehi Mat. Nauk 14 (1959), no. 5 (89), 45-96. German translation: "Endlichkeitsbedingungen in der Gruppentheorie", (VEB Deutscher Verlag der Wissenschaften, Berlin, 1963).

[1] Charles W. Curtis; Irving Reiner, Representation theory of finite groups and associative algebras (Pure and Applied Mathematics, $X I$; Interscience, [John Wiley \& Sons], New York, London, 1962).

[8] B. Eckmann und A. Schopf, "Über injektive Moduln", Arch. Math. 4 (1953), 75-78.

[9] A.D. Gardiner, B. Hartley and M.J. Tomkinson, "Saturated formations and Sylow structure in locally finite groups", J. Algebra (to appear).

[10] B. Hartley, "Sylow subgroups of locally finite groups" Proc. London Math. Soc. (to appear).

[11] Lawrence Levy, "Torsion-free and divisible modules over non-integral-domains", Canad. J. Math. 15 (1963), 132-151.

[12] L.G. Kovács and M.F. Newman, "Direct complementation in groups with operators", Arch. Math. 13 (1962), 427-433.

[13] D. McDougall, "The subnormal structure of some classes of soluble groups", J. Austral. Math. Soc. (to appear).

[14] D. McDougalI, "Soluble groups with the minimal condition for normal subgroups", Math. 2. (to appear).

[15] Tsai Chi-Te, Report on injective modules (Queen's papers in pure and applied mathematics, no. 6; Queen's University, Kingston, Ontario, 1966).

[16] John S. Wilson, "Some properties of groups inherited by normal. subgroups of finite index", Math. Z. 114 (1970), 19-21.

University of Warwick,

Coventry, England;

Australian National University,

Canberra, ACT. 